\title{
LITERATURA ENTRE LENGUAS: EL CONFLICTO LINGÜÍSTICO EN EL AMANTE BILINGÜE DE JUAN MARSÉ
}

\author{
LITERATURE BETWEEN LANGUAGES: LINGUISTIC CONFLICT IN \\ EL AMANTE BILINGÜE BY JUAN MARSÉ
}

\author{
Marco Neves \\ CETAPS / Universidade Nova de Lisboa \\ Portugal \\ marconeves@gmail.com
}

\begin{abstract}
Resumen: "Creo que la patria de un escritor ni siquiera es la lengua, es el lenguaje."1 La frase de Juan Marsé sorprenderá a muchas víctimas de los simplismos dominantes, pues se basa en ideas muy diferentes a ciertas "mentiras piadosas" sobre lo que es la literatura, de lo que está hecha esa literatura y cómo se relaciona con la nación y la lengua. En este artículo, nos centramos en una herida abierta en la relación entre lengua, literatura y nación: la situación lingüística de Cataluña. Sin embargo, las conclusiones pueden ser útiles para realizar análisis más amplios en el ámbito hispano, ibérico y de los Estudios Literarios. El foco de nuestro análisis será la novela de Juan Marsé El amante bilingüe. Describiremos algunas ideas simplificadas sobre la relación entre la lengua, la literatura y la nación y veremos cómo estas ideas son negadas en la novela de Marsé.
\end{abstract}

Palabras clave: Lenguaje, conflicto, novela, literatura catalana, literatura española, nacionalismo.

\begin{abstract}
I think a writer's homeland isn't a specific language, but rather language in general." Juan Marsé’s sentence will surprise many victims of simplistic ideas, because it is very far from certain "pious lies" about what literature is, what it is made of and how it relates to the ideas of nation and language. In this article, we focus on an open wound in the relationship between language, literature and nation: the linguistic situation in Catalonia. However, conclusions may be useful for further studies in the Hispanic, Iberian and Literary Studies spheres. The focus of our analysis will be Juan Marsé's novel El amante bilingüe. We will describe some simplified ideas about
\end{abstract}

${ }^{1}$ Traducción de "Creio que a pátria de um escritor não é sequer a língua, é a linguagem.” - Entrevista con Juan Marsé, Visão, 10 de mayo de 2012. 
the relationship between language, literature and nation and see how these ideas are denied by Marsé's novel.

Keywords: Language, conflict, novel, Catalan literature, Spanish literature, nationalism.

Recibido: 18/11/2019. Aceptado: 04/06/2020.

\section{La sacralización de la lengua}

$\mathcal{H}$ l amante bilingüe provoca al lector -y lo provoca a atacar un fenómesociedades como la catalana-. Este fenómeno puede llamarse la sacralización de la lengua nacional ${ }^{2}$. Tal vez sería de esperar que un escritor sacralizase, o al menos respetase, el material con el que trabaja. Sin embargo, esto es exactamente lo que Juan Marsé nos dice en la frase citada en el epígrafe: el material del novelista es el lenguaje, no una lengua cualquiera. A veces incluso tendrá que maltratar la lengua (según los criterios de los nacionalistas puristas) para crear y provocar al lector.

Esta sacralización de la lengua está ligada a una identificación entre nación y lengua por un lado y lengua y literatura por otro, lo que conduce a una identificación, en la mente de los nacionalistas, entre la literatura nacional y la "esencia de la nación", una identificación que se hace a través de la lengua. Esto se debe a que la lengua está indeleblemente ligada a la esencia de la nación, imbuida de su historia, según esta ideología ${ }^{3}$.

Por otro lado, la lengua tiene una forma pura y varias formas "impu-

${ }^{2}$ Se trata de nacionalismo lingüístico, analizado en profundidad por Cabrera (2008).

${ }^{3}$ No nos olvidemos de las palabras de Dámaso Alonso, que expresan esta idea tan claramente: "Oh, no!: no ha sido casualidad que las primeras frases francesas que conservamos sean militares y políticas (genio de Richelieu, glorias de Austerlitz). Ni que las primeras italianas miren a los bienes materiales (recuérdense las burlas contra banqueros genoveses, en nuestras letras clásicas, pero no se olvide tampoco cuánto oro de Venecia hay en los cuadros de Tiziano). Y no puede ser azar, no. O, si acaso lo es, dejadme esta emoción que me llena al pensar que las primeras palabras enhebradas en sentido, que puedo leer en mi lengua española, sean una oración temblorosa y humilde. El César bien dijo que el español era lengua para hablar con Dios. El primer vagido del español es extraordinario, entre los de sus hermanas. No se dirige a la tierra: con Dios habla, y no con los hombres." (Alonso, 1964) 
ras”. Si sabemos que la norma está definida por la convención social, el nacionalismo lingüístico -el término preciso con el que podemos definir tal sacralización de la lengua nacional- cree que la norma es la expresión más refinada del espíritu nacional, la superviviente de un proceso de selección que conduce a la purificación de la lengua y a su elevación al estatus de lengua nacional. La coincidencia que vemos entre la norma y ciertas clases dominantes y regiones de importancia económica y política es un fenómeno que no altera en absoluto la certeza casi religiosa de quienes creen que la norma es la lengua tal como debe hablarse ${ }^{4}$. Esto justifica actitudes racistas cuasi sociales basadas en la variación lingüística, basadas en la necesidad patriótica de defender la lengua. Después de todo, la lengua, como parte del "espíritu" de una nación, no se adecúa a análisis científicos, sino que tiene su propio sacerdocio y sus propias revelaciones.

En resumen: para los nacionalistas, la lengua es la nación. En el exterior, la lengua distingue a cada nación de las naciones vecinas. Internamente, debe ser uniforme y cualquier ataque a esta uniformidad es un ataque a la nación. En otras palabras, la lengua es uno de los elementos centrales en la configuración de la identidad de cualquier nación, tanto en su diferenciación del exterior como en la unidad interna que se busca. Este es un punto central en cualquier ideología nacionalista más o menos marcada ${ }^{5}$.

Hemos señalado anteriormente que la lengua, como entidad indeleblemente ligada al espíritu de la nación, tiene su propio sacerdocio -este sacerdocio incluye, en opinión de los nacionalistas, a los escritores-. La función esencial de un escritor sería defender y refinar la lengua. Así, la literatura nacional será siempre una literatura nacionalista, porque defiende, con el uso de la lengua nacional, la propia nación y sus valores, inscritos en la lengua.

La literatura también será un espacio de afirmación de la nación en competencia con las demás naciones. En esta secuencia, se entiende que la

${ }^{4}$ La defensa de la norma puede adoptar una versión “débil” -y algo legítima- que considera que la norma debe ser defendida para permitir la comunicación interna en un país -sin embargo, esta defensa asume la norma como una convención necesaria. Luego está la defensa "fuerte" - y en cuanto a nosotros ilegítima - que cree en la norma como la versión más perfecta de la lengua- la más perfecta, la más lógica, la más racional.

${ }^{5}$ No está en nuestro análisis estudiar el origen de esta idea, que estará, de forma familiar, en el proceso de desarrollo de los Estados nacionales europeos en los últimos 200 años. 
literatura es vista, de manera simplista, como un espacio donde la lengua debe ser el modelo más puro y elevado de la norma -y también se entiende que, para los nacionalistas, el uso "desviado" de la lengua en los buenos escritores es extraño- ${ }^{6}$.

Todo esto tiene sus raíces en un profundo malentendido de lo que es la literatura y, específicamente, la novela. No es que la literatura y la novela no puedan ser utilizadas con fines nacionalistas. Pero, en realidad, la novela es un género profundamente democrático y no se entrega a ideologías estrechas como el nacionalismo (lingüístico o de otro tipo). La novela no se basa en una lengua, en una norma, sino que consiste en un tejido formado por varios lenguajes opuestos, como nos dice Bakhtin (2004: 261-262)7, para quien la novela es un espacio donde están presentes varias lenguas y lenguajes:

The novel as a whole is a phenomenon multiform in style and variform in speech and voice. In it the investigator is confronted with several heterogeneous stylistic unities, often located on different linguistic levels and subject to different stylistic controls. [...] The stylistic uniqueness of the novel as a genre consists precisely in the combination of these subordinated, yet still relatively autonomous, unities (even at times comprised of different languages) into the higher unity of the work as a whole: the style of a novel is to be found in the combination of its styles the language of a novel is the system of its "languages." (destaque nuestro)

El escritor utiliza el lenguaje, no la lengua abstracta defendida por cualquier nacionalismo lingüístico. La novela es un lienzo pintado con diferen-

\footnotetext{
${ }^{6}$ Curiosamente, esta idea se alimenta de una falacia: los nacionalistas creen que la buena literatura utiliza la buena norma de la lengua, apuntando a los "grandes clásicos" como prueba -clásicos que obviamente se sitúan en un tiempo pasado (porque uno de los dogmas del nacionalismo es el de la decadencia de la Nación)-. Ahora bien, en ese mismo período, las mismas obras consideradas hoy como canónicas serían vistas como "subversivas". En otras palabras, los nacionalistas toman ciertas obras, definen la norma basada en ellas y terminan señalando las obras como ejemplos del uso de la norma, que es un argumento circular. Por otro lado, las obras actuales que se apartan de la norma son vistas con recelo - por ejemplo, los diversos ataques a Saramago por la forma "incorrecta" en que escribe forman parte de esta identificación entre la norma (una mera convención) y la necesidad de defender a la Nación-.

${ }^{7}$ La cita está en inglés porque esta fue la traducción que consultamos para este trabajo. Por la misma razón, usamos la forma "Bakhtin" para transliterar el apellido del autor.
} 
tes lenguajes, puestos en oposición, desensamblando en esta oposición el propio posicionamiento absoluto que cada uno cree tener en relación con el mundo. El autor tiene una cierta posición y trata de mostrar al lector una cierta perspectiva del mundo, pero esta perspectiva es plural, polifónica. Esto, por supuesto, en ciertas novelas, las novelas que podríamos llamar bakhtinianas.

\section{La desacralización de la lengua}

El amante bilingüe es la negación de la idea nacionalista de identificación exacta entre territorio, nación, lengua, norma y literatura; esta obra desestabiliza y rompe las ilusiones nacionalistas enfrentadas en Barcelona. De hecho, una visión que parte del nacionalismo lingüístico catalán entiende la lengua catalana como propia de la ciudad, como la única realmente legítima -y defiende una literatura en una norma específica de esa lengua-. Del mismo modo, un nacionalismo lingüístico español defenderá el uso del español en la literatura y siempre se siente amenazado por el uso del catalán o de variantes menores de la lengua, como el acento andaluz. Porque la literatura de Juan Marsé trata del uso real del lenguaje en la ciudad, tomando este material y moldeándolo de forma artística, en una novela satírica y provocadora. En cierto modo, abandona el nivel de artificialidad implícito en una novela solo en catalán o en una novela solo en español que tiene como escenario Barcelona, donde el contacto entre las lenguas, entre las distintas variantes de las dos lenguas, entre las normas y las desviaciones, es una constante.

En una sociedad como la catalana, el poder de atracción ejercido por dos “centros lingüísticos” es evidente: el español y el catalán. La ideología nacionalista catalana siente la necesidad de una normalización lingüística, siente una especie de "anhelo" por una época mítica en la que el catalán sería la única lengua de una nación única. Por otro lado, la ideología nacionalista española considera el catalán como una amenaza a su utopía de una "España única", con una lengua, un territorio, una nación. Si la misma situación sobre el terreno niega la validez de cualquiera de estas perspectivas como ideologías totalitarias, la fuerza de estas dos ideologías se siente claramente en esta compleja sociedad. 
Por otro lado, la presencia de dos lenguas en Cataluña muestra cómo la situación lingüística de un pueblo no es solo una regla y posibles desviaciones de la misma. El lenguaje se divide en lenguas, dialectos, sociolectos, registros, jerga profesional. La variación lingüística es un hecho, es la base, la realidad que encontramos en las calles de Barcelona, donde una persona mezcla dos lenguas en la misma frase con sus amigos, usa un catalán "puro" en una situación determinada, entra en un diálogo en español en otra situación y así sucesivamente.

El catalán y el español, como normas defendidas por nacionalismos opuestos, son abstracciones. En las calles de Barcelona, se encuentran, se mezclan en las mismas frases, se enfrentan. Como menciona uno de los personajes de El amante bilingüe, las dos lenguas están "en contacto vivo y caliente con el individuo" (p. 109). Otro personaje, en el mismo diálogo, se refiere al punto en que "las dos lenguas se friccionan" (p. 109).

Las sugerencias sexuales son obvias y significativas. La confrontación y el contacto entre lenguas se ve en paralelo a la confrontación y el contacto sexual entre personajes. La hibridez, el deseo del otro, la necesidad de dejar atrás las máscaras demasiado perfectas y bajar a la tierra, tiene paralelo en la necesidad sexual del otro. Las lenguas puras son básicamente las lenguas imaginadas y deseadas platónicamente por los nacionalistas lingüísticos de todos los ámbitos. El lenguaje real, usado y abusado todos los días, es el verdadero material de la verdadera literatura.

Norma, la mujer de Joan Marés, que lo traiciona con un andaluz (un charnego, un murciano), tiene un nombre significativo en su relación con la lengua. Es una sociolingüista que trabaja para la Generalitat en el departamento responsable de la normalización de la lengua catalana. Así como Marés le cuenta al amante de Norma cuando se conocieron, Norma “[e]studiaba filología catalana en la universidad y era una chica romántica y progre" (p. 17). A pesar de ser "progre", seguía formando parte de la burguesía catalana, con vínculos más que sospechosos con el régimen franquista: "Norma era hija única del difunto Víctor Valentí, fabricante de cinturones de cuero y artículos de piel que en los años cuarenta hizo una fortuna al obtener contratos en exclusiva del ejército" (p. 14).

Este personaje normativo, que trabaja por la lengua catalana, tratando de purificar la sociedad de los elementos extranjeros representados por el castellano, desea, en el fondo, a ese otro que rechaza durante el día: el 
amante andaluz, encarnación del estereotipo del inmigrante "charnego". Como dice Marés:

Cuando empecé a sospechar que Norma me engañaba, pensé en Eudald Ribas o en cualquier otro señorito guaperas de su selecto círculo de amistades, pero no tardé en descubrir que su debilidad eran los murcianos de piel oscura y sólida dentadura. (p. 11)

Joan Marés, que había asumido la más pura identidad catalana para complacer a la mujer, se confunde: para recuperar a la mujer, establece entonces un plan de transfiguración en Faneca, un murciano que se ajusta a la fantasía hasta entonces desconocida de su ex mujer. El personaje principal navega entre Joan Marés ${ }^{8}$ y Faneca (nombre de un amigo de la infancia). Al final, la identidad catalana abandona la escena y nos quedamos con Faneca quien, sin embargo, no puede permanecer establemente en una determinada máscara. El protagonista no puede definirse y es la encarnación de una identidad nacional y lingüística híbrida, indefinida y, supongamos, más real, en una ciudad donde una misma frase puede cruzar lenguas e identidades.

En definitiva, Juan Marsé utiliza el material lingüístico de que dispone para crear una construcción artística basada claramente en lo que se puede llamar una "novela bakhtiniana" (una novela que basa su interpretación en el uso de diferentes lenguajes y lenguas por parte del lector). Marsé analiza la singular esquizofrenia lingüística catalana y denuncia todas las soluciones que pretenden "limpiar" la situación, purificar la lengua, excluir las otras identidades que subsisten en la ciudad.

Ahora se entiende claramente lo que Juan Marsé quiere decir cuando afirma que la patria de un escritor es el lenguaje. Marsé crea su obra utilizando el lenguaje, este magma lingüístico humano, en constante variación, que solo las ideologías simplistas pueden resumir en la existencia de lenguas discretas y uniformes, como si cada persona, cada nación, cada ciudad solo pudiera pertenecer a un sistema lingüístico -y la literatura tuviera que seguir este esquema demasiado reductor-.

El amante bilingüe provoca así al lector en su presunta identidad defi-

\footnotetext{
${ }^{8}$ Nótese la interesante alusión al propio nombre del autor.
} 
nida. Ironiza a Norma y su deseo de normalización (lingüística y nacional) ${ }^{9}$, que esconde un deseo sexual del otro, de quien se aleja de esta visión estéril de la lengua y de la sociedad. También ironiza a Joan Marés, que siente la necesidad de transformarse en Faneca ${ }^{10}$. Provoca al lector castellano, con el uso del catalán y del español andaluz, pero provoca aún más al lector catalán, para quien el mero hecho de escribir en español en Cataluña tiene claras implicaciones. Pero Juan Marsé no elige un campo: utiliza el lenguaje -español, catalán, español andaluz, todo en la misma obra, a veces en el mismo párrafo-. La patria del escritor es el lenguaje, no una lengua cualquiera. Hay aquí un impulso democrático, una huida de identidades demasiado definidas, una necesidad de afirmarse como un híbrido, incoherente, roto por dentro, pero genuino y verdadero, y esta necesidad se aplica al personaje, a la ciudad, y probablemente al autor.

Es también, por supuesto, una invitación al lector a dejar su capullo ideológico, sus certezas lingüísticas, nacionalistas y normativas. El objetivo es evitar que el lector caiga en la trampa del nacionalismo lingüístico.

Juan Marés nos muestra un paisaje lingüístico de Barcelona, con todas las implicaciones que este paisaje tiene en términos de identidad, conflicto, indefinición o demasiada definición -es a partir de este magma que se crea la literatura, compuesta por varios lenguajes, lenguas, hablas- de una realidad cruda, utilizada como material para la literatura, tratada artísticamente, pero que no necesariamente convive con ideologías como el nacionalismo lingüístico, por muy "naturales" que puedan parecer.

Es en este contexto que se comprende la genialidad del último párrafo de la obra, cuando Faneca olvida cualquier identidad pura y mezcla con impunidad el catalán y el español andaluz:

Pué mirizté, en pimé ugá me'n fotu e menda yaluego de to y de toos i així finson vostè vulgui poque nozotro lo mataore catalane volem toro catalane, digo, que menda s'integra en la Gran Encisera hata onde le dejan

\footnotetext{
${ }_{9}$ Algunos de los pasajes más interesantes son los diálogos entre Joan Marés transformado en Faneca, por teléfono, con Norma, tratando de entender los términos correctos en catalán para su uso en el inexistente negocio de Faneca -la crítica al proceso de normalización de la lengua catalana basada en la imposición es obvia-.

${ }^{10}$ El anagrama del nombre revela, probablemente, cómo esta pregunta está muy presente en la piel de este autor.
} 
y hago con mi jeta lo que buenamente puedo, ora con la barretina ora con la montera, o zea que a mí me guta el mestizaje, zeñó, la barreja y el combinao, en fin, s'acabat l'explicació i el bròquil, echusté una moneíta, joé, no sigui tan garrapo ni tan roñica, una pezetita, cony, azí me guta, rumbozo, vaya uzté con Dió i passiu-ho bé, senyor... (p. 220)

Volviendo al principio de la obra: cuando Marés entra en la habitación donde descubre al amante de la mujer, ve, en primer lugar, su propia imagen en el espejo -y luego al amante de la mujer-. Hay aquí un primer indicio del despliegue de la identidad del protagonista -y de su origen: el protagonista no puede verse en el espejo como una imagen artificial de un catalán puro, que ha perdido, aun así, a la mujer- que se convierte en el otro, en el que piensa que es deseado por la mujer, terminando por perderse como una identidad fija.

Este rechazo de una identidad única después del abandono de la mujer se refleja en el rechazo de una sola lengua o, más bien, en la aceptación de la verdadera hibridación de la identidad nacional y lingüística. Al final, Marés es un "charnego" con "su repertorio de sardanas y de canciones populares catalanas", tocando junto a la Sagrada Familia para que los turistas lo vean.

En conclusión, como demuestra Juan Marsé, la literatura no se conforma con divisiones artificiales y visiones totalitarias de la realidad. Este análisis es un buen lugar para preguntarnos: ¿es legítimo separar la literatura por simples criterios nacionales? ¿No es Juan Marsé un escritor catalán? Obviamente, lo es. Y también español. Escribe en español, pero lo mezcla con el catalán, enfrentando las dos lenguas. Además, utiliza un español andaluz, mezcla varios registros, etc.

Podemos dividir la literatura según un criterio nacional, pero solo será uno de los varios criterios que podemos utilizar. Contrariamente a lo que se puede decir, podemos hacer análisis legítimos utilizando otros criterios. La "literatura catalana" será una división legítima, al igual que la "literatura en catalán", la "literatura española", la "literatura en castellano", la "literatura catalana en castellano", etc. Por supuesto, las etiquetas nacionales tienen una gran fuerza en la sociedad y no debemos olvidar este hecho cuando analicemos la literatura. Forman parte del paisaje cultural donde se inscriben las obras. Para una discusión de los criterios de demarcación de las literaturas nacionales, ver Guillén (1998).

Una ideología nacionalista puede defender de manera absoluta la idea 
de que la literatura está vinculada a una nación en particular. Las mismas obras literarias pueden estar imbuidas de esta ideología -entonces se tratará de una literatura nacionalista-. Sin embargo, la literatura, tal como la practican autores como Juan Marsé, no encaja con esta inscripción absoluta en una ideología específica: el nacionalismo.

La literatura es una forma de arte que utiliza el lenguaje como material de construcción artística. Después de todo, si el material utilizado en la novela fuera algún tipo de norma lingüística (a defender con uñas y dientes), el último párrafo de El amante bilingüe sería un desastre. De hecho, es un ejemplo acabado del complejo, irónico y satírico tejido que un buen novelista crea, utilizando los distintos lenguajes, lenguas e identidades de una sociedad para construir una novela, donde todas estas fuerzas se enfrentan y, al mismo tiempo, se combinan para deleitar e instruir al lector, que estará mucho menos sujeto a ideas simplistas, nacionalistas y totalitarias de la literatura y de la vida.

\section{Referencias bibliográficas}

Alonso, Dámaso (1964). De los siglos oscuros al de Oro. Madrid: Gredos. Bakhtin, M. M. (2004). The Dialogic Imagination. Ed. Michael Holquist. Austin: University of Texas Press.

Cabrera Moreno, Juan Carlos (2008). El nacionalismo lingüístico. Una ideología destructiva. Barcelona: Península.

Guillén, Claudio (1998). Múltiples moradas. Barcelona: Tusquets.

Marsé, Juan (1990/2004). El amante bilingüe. Barcelona: Editorial Planeta. 